\title{
Valor del curetaje endocervical en el diagnóstico de neoplasias cervicales: Instituto Materno Infantil de Bogotá. 1986-1988
}

\author{
Dres. Carlos Alberto Díaz Rodríguez*, Miguel Eduardo Aragón Sánchez**
}

\begin{abstract}
RESUMEN. Se revisaron 202 historias clínicas de pacientes que acudieron a la consulta de oncología del I.M.I. y que fueron intervenidas entre los años de 1986 a 1988 (cono o histerectomía).

El objetivo principal consiste en evaluar el resultado del CEC (curetaje endocervical), comparándolo con la citología, la biopsia exocervical y la pieza final de anatomía patológica con el fin de valorar el verdadero papel de este método diagnóstico para NIC.

Encontramos un resultado positivo del CEC en $55 \%$ de casos con colposcopia satisfactoria y un $60 \%$ de los casos con colposcopia insatisfactoria. El CEC aumenta la agudeza diagnóstica en sólo $6 \%$ de casos con colposcopia insatisfactoria.

La sensibilidad del CEC corresponde a un $64 \%$ y el valor predictivo positivo del $72 \%$.

Los falsos negativos encontrados corresponden al 36\%. EL CEC no aportó el diagnóstico de invasión.

Estos resultados se comparan con los resultados encontrados en otros estudios. (Rev Col Obstet Ginecol 1991; 42(3): 209-217).
\end{abstract}

PALABRAS CLAVES: Curetaje endocervical, neoplasia intracervical (NIC).

SUMMARY. From the revision of 202 clinical histories of the patients who assisted to the oncology clinical in the Materno-Infantil Institute and the one's who have a surgical intervention between 1986 and 1988.

Was made with the main objective to evaluate the CEE (endocervical curettage) as a diagnostical method for cervical intraepithelial neoplasia, in comparission with the citology, biopsy exocervical and the final pathology-anatomy instrument.

The results were positives in the $55 \%$ of the cases with a satisfactory colposcopy and $60 \%$ of the cases with a unsatisfactory colposcopy. The CEE only shows a better diagnostic spectrum in $6 \%$ of the cases with a unsatisfactory colposcopy.

The CEE sensibility is $64 \%$ and the positive predicted value is $72 \%$.

The false negative cases were of $36 \%$ and the CEE did not give an invasion diagnostic.

The final results of this study were compared with the results of other studies.

KEY WORDS: Endocervical curettage, cervical intraepithelial neoplasia (CIN).

\section{Introducción}

En la literatura mundial se reportan estadísticas muy variables en cuanto a la especificidad y sensibilidad del CEC, así como una variable enorme en cuanto a falsos positivos y negativos $(1,2,4,5,6,915,17,18,19)$.

Como hemos visto, existe controversia en cuanto al uso del CEC, y va desde aquellos que no lo usan hasta aquellos que lo usan en todos los casos $(11,13,17,18,19)$.

También existe discrepancia en cuanto al uso si la colposcopia es satisfactoria o insatisfactoria $(11,13)$.

Algunos dicen que el uso del CEC disminuye la cantidad de conos en el $76 \%$ de los casos (13) y otros dicen que el $\mathrm{CEC}(+)$ fue la mayor razón para realizar cono en el $58 \%$ de las pacientes $(12,20)$.

La polémica va más allá cuando un grupo atribuye al CEC un gran valor cuando existe invasión (13), mientras otros dicen que no excluye la posibilidad de encontrar un ca. invasivo $(11,13)$.

\footnotetext{
* Residente de tercer año, Departamento de Ginecobstetricia.

** Profesor Asistente. Jefe Sección de Oncología I.M.I., Depto. de Ginecobstetricia, Universidad Nacional. Instituto Materno Infantil. Santafé de Bogotá, D.C. Carrera 10 No. 1-99.
}

Otros refieren que en colposcopia insatisfactoria todos deben tener CEC (11) mientras un grupo refiere que en colposcopia insatisfactoria no se debe o no se necesita el CEC, pues todos van a cono (12).

La discrepancia de opiniones se acentúa por el informe de algunos de que los falsos positivos del CEC se deben a contaminación en la muestra con el exocervix y que el resultado depende del volumen de dicha muestra $(15,16)$. Otros se limitan a darle importancia al CEC de acuerdo a la terapéutica que se vaya a seguir con la paciente (13).

Por otro lado, en cuanto a los esquemas de diagnóstico de NIC, existen algunos que no incluyen al CEC y otros que basan la terapéutica en lo encontrado en dicho examen $(3,8)$.

Con lo anteriormente expuesto, vemos que existen múltiples opiniones acerca del uso o deshuso del CEC, sin llegar a una verdadera conclusión.

Es de notar que la revisión de cono en el I.M.I. entre 1985-1988 (20) el 38.5\% de indicaciones correspondió a compromiso endocervical (indicación de cono diagnóstico) y en total se realizaron $47 \%$ de conos por indicación, debido a compromiso endocervical. 
Con base en los anteriores datos es difícil establecer una hipótesis de estudio y sólo nos referiremos a los factores dados por Andersen (16) que soportan la necesidad de realizar un CEC:

1. El CEC puede mostrar una lesión mayor que la del exocervix.

2. El CEC puede mostrar un adenocarcinoma.

3. A veces el único dato positivo en el esquema diagnóstico es el CEC.

4. En colposcopia insatisfactoria el CEC puede ayudar a establecer la lesión, aunque necesariamente se necesite cono.

Podemos resumir los datos obtenidos por los diferentes autores en las siguientes tablas:

En la Tabla 1 se correlaciona la positividad del curetaje de acuerdo a la colposcopia.

En la Tabla 2 se correlaciona la frecuencia en la cual el CEC detecta mayor lesión que la biopsia de exocervix de acuerdo a si la colposcopia es satisfactoria o insatisfactoria, según los diferentes autores.

En cuanto a las tasas de falsos positivos, negativos y frecuencia de material insuficiente según las diferentes revisiones, se dan en las tablas 3,4 y 5 , respectivamente.

El valor de la prueba del CEC, según Andersen, se encuentra en la Tabla 6.

Tabla 1

POSITIVIDAD DEL CEC DE ACUERDO A LA COLPOSCOPIA

\begin{tabular}{|c|c|c|c|}
\hline Colposcopia Satisfactoria & $\%$ & Colposcopia Insatisfactoria & $\%$ \\
\hline Hatch y col. $(11,14)$ & 13.9 & & 52.7 \\
\hline Drescher y col. $(11,14)$ & 17.9 & & 48.7 \\
\hline Urcuyo y col. $(11,14)$ & 8.6 & & 57.3 \\
\hline Wetrich y col. (13) & 7.0 & & 34.0 \\
\hline Moseley y col. (12) & 10.6 & & 45.0 \\
\hline Spirtos y col. (14) & 4.7 & & \\
\hline Saltzman y col. (14) & 10.0 & & \\
\hline
\end{tabular}

Tabla 2

FRECUENCIA EN LA CUAL EN CEC SE DETECTA LESION MAYOR QUE LA ENCONTRADA

EN LA BIOPSIA DE EXOCERVIX DE ACUERDO AL TIPO DE COLPOSCOPIA: (CEC > BX)

\begin{tabular}{|lrrr|}
\hline Colposcopia: & Satisfactoria \% & Insatisfactoria \% & Lesión no Vista \% \\
\hline Hatch y col. (11) & $1.2 \%$ & $15.7 \%$ & $30.5 \%$ \\
Drescher y col. (11) & $3.8 \%$ & $21.1 \%$ & \\
Moseley y col. (12) & $13.0 \%$ & $3.0 \%$ & \\
\hline
\end{tabular}

Tabla 3

FRECUENCIA DE FALSOS NEGATIVOS DEL CEC (EN COLPOSCOPIA INSATISFACTORIA)

\begin{tabular}{|lr|}
\hline Estudio & $\%$ \\
\hline Spirtos y col. (14) & 24 \\
Urcuyo y col., Jafari y col., Hatch y col. (11,13) & $41-45$ \\
Andersen y col. (16) & 45 \\
\hline
\end{tabular}

(Hatch y col. encontraron un porcentaje de positividad del CEC en $62.5 \%$ de colposcopias en las cuales la lesión no se visualizó) (11).

Tabla 4

FRECUENCIA DE FALSOS POSITIVOS DEL CEC

\begin{tabular}{|lr|}
\hline Estudio & $\%$ \\
\hline Spirtos y col. (14) & 4.7 \\
Andersen y col. (16) & 25.0 \\
\hline
\end{tabular}

Los autores están de acuerdo que esto se debe a contaminación de la muestra al paso por el exocervix.

Tabla 5

FRECUENCIA DE MATERIAL INSUFICIENTE EN CEC

\begin{tabular}{|lr|}
\hline Estudio & $\%$ \\
\hline Spirtos y col. (14) & 6.5 \\
Wetrich y col. (13) & 14.4 \\
Andersen y col. (16) & 30.6 \\
\hline
\end{tabular}

Tabla 6

VALOR DE LA PRUEBA (CEC) SEGUN ANDERSEN (16)
Valor predictivo negativo

\section{Material y métodos}

Se estudiaron las pacientes tratadas en la Clínica de Oncología del I.M.I. entre los años de 1986-1988 a quienes se les practicó CEC y posteriormente tratamiento definitivo.

Se compara el resultado del CEC con la biopsia y la pieza final, estableciendo la diferencia en cuanto a si la colposcopia fue satisfactoria o insatisfactoria.

Para establecer el resultado de sensibilidad, especificidad y valor predictivo, así como falsos positivos y negativos, se compara el resultado del CEC en relación con la pieza final de anatomía patológica, estableciendo si la invade o no el canal (la lesión).

Los resultados se colocarán en una tabla de 4 casillas (tabla de $2 \times 2$ ).

Para establecer en cuántos casos el CEC fue mayor, en cuántos igual y en cuántos menor que el resultado de la biopsia o la pieza quirúrgica, se discriminan en tablas diferentes todas las opciones diagnósticas de NIC.

Las variables, edad, paridad y citología, se tomarán independientemente de los resultados y sólo servirán de descripción de la muestra.

Se usó la prueba del $\mathrm{Ji}^{2}$ para significación estadística.

Se define como CEC positivo aquel que el patólogo reporta como muestra adecuada, con evidencia de displasia grado I o mayor, o franca neoplasia.

El CEC negativo incluye los casos que el patólogo reporta 
como negativo para neoplasia, endocervicitis crónica y agu$\mathrm{da}$, metaplasia escamosa madura o inmadura, endocervicitis polipoide, muestras con atipias leves o severas, o simplemente endocervix normal.

Se define colposcopia satisfactoria cuando se observan todos los límites de la zona de transformación en toda su extensión y con evidencia de sus bordes.

Colposcopia insatisfactoria se define aquella en que no se observan los límites de la zona de transformación, pues aquella se introduce profundo en el canal.

Se define como citología positiva: comprende las citologías A3-A4 -A5.

Citologías negativas: incluyen A2-A2 con atipias leves o severas.

Para el reporte de células endocervicales en la citología se sigue el mismo parámetro, encontrando que citología negativa comprende las B1-B2 y positivas aquellas con reporte B3 o mayor.

Las biopsias exocervicales tomadas en este estudio son totalmente biopsias dirigidas bajo colposcopia.

El resultado de la muestra obtenida en el CEC se reportó como abundante moderado o escaso material de acuerdo con los criterios que rigen en nuestro departamento de patología.

\section{Resultados}

De acuerdo a los parámetros expuestos se revisaron 202 casos de pacientes atendidas en la Clínica de Oncología del Instituto Materno Infantil de Bogotá a quienes se estableció tratamiento mediante conización o histerectomía y que tenían por lo menos un CEC.

El resultado del CEC fue el siguiente:

- CEC no apto: 57 casos (28.2\%), distribuidos así:

- 44 con muestra insuficiente.

- 13 se reportó endometrio.

- CEC apto: 145 casos (71.8\%). Estas pacientes son las que incluimos en el estudio. Los casos se distribuyen así: Con un solo CEC: 101 pacientes.

Con dos (2) CEC, uno de los cuales no fue apto, 22 pacientes. Con dos (2) CEC, aptos: 22 pacientes.

- En una de estas pacientes no se realizó citología.

- No se realizó biopsia exocervical en un caso.

- No se realizó colposcopia en dos casos.

Edad: La distribución de la edad para las pacientes se encuentra en la Tabla 7 (Gráfica 1).

Se encontró un promedio de edad $(\bar{X})$ de 40.9 años, con una media (Me) de 39.7 años.

Paridad: La distribución de las pacientes por paridad se encuentra en la Tabla 8 (Gráfica 2).

Resultado del CEC: En cuanto a positividad o no del CEC, encontramos lo expuesto en el Cuadro 1.

De los 62 casos reportados como CEC negativo los patólogos informan escaso material o fragmento en 17 casos (27.4\%).

Comparación del resultado del CEC y la citología: El resultado del CEC comparado con la positividad de la citología para células escamosas se presenta en el Cuadro 2.

De las 144 citologías sólo en $62(43 \%)$ se reportan células endocervicales.
La comparación del resultado del CEC frente a la citología para células endocervicales se reporta en el Cuadro 3. Hacemos notar que todos los casos de citología positiva endocervical fueron B3.

Tabla 7

DISTRIBUCION POR EDAD

\begin{tabular}{|lrr|}
\hline Edad & No. Casos & $\%$ \\
\hline $20-24$ & 1 & 0.7 \\
$25-29$ & 17 & 11.7 \\
$30-34$ & 20 & 13.8 \\
$35-39$ & 36 & 24.8 \\
$40-44$ & 31 & 21.4 \\
$45-49$ & 15 & 10.3 \\
$50-54$ & 14 & 9.6 \\
$55-59$ & 2 & 1.4 \\
60 o más & 9 & 6.2 \\
\hline \multicolumn{2}{|c|}{ TOTAL } & 145 \\
\hline
\end{tabular}

$\overline{\mathrm{X}}=40.9$ años

$\mathrm{Me}=39.7$ años

Tabla 8 DISTRIBUCION POR PARIDAD

\begin{tabular}{|c|c|c|}
\hline Paridad & No. Casos & $\%$ \\
\hline $\begin{array}{l}0 \\
1 \\
2 \\
3 \\
4 \\
5 \text { o más }\end{array}$ & $\begin{array}{r}1 \\
8 \\
10 \\
25 \\
17 \\
84\end{array}$ & $\begin{array}{r}0.7 \\
5.5 \\
6.9 \\
17.2 \\
11.7 \\
57.9\end{array}$ \\
\hline TOTAL & 145 & 100.0 \\
\hline
\end{tabular}

Gráfica 1

DISTRIBUCION DE CASOS POR EDAD

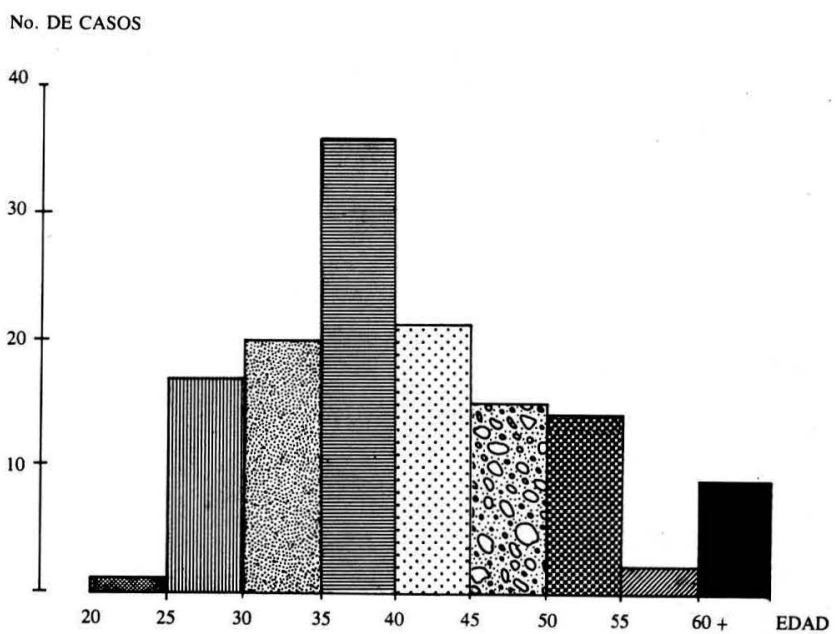


Gráfica 2

DISTRIBUCION DE CASOS POR PARIDAD

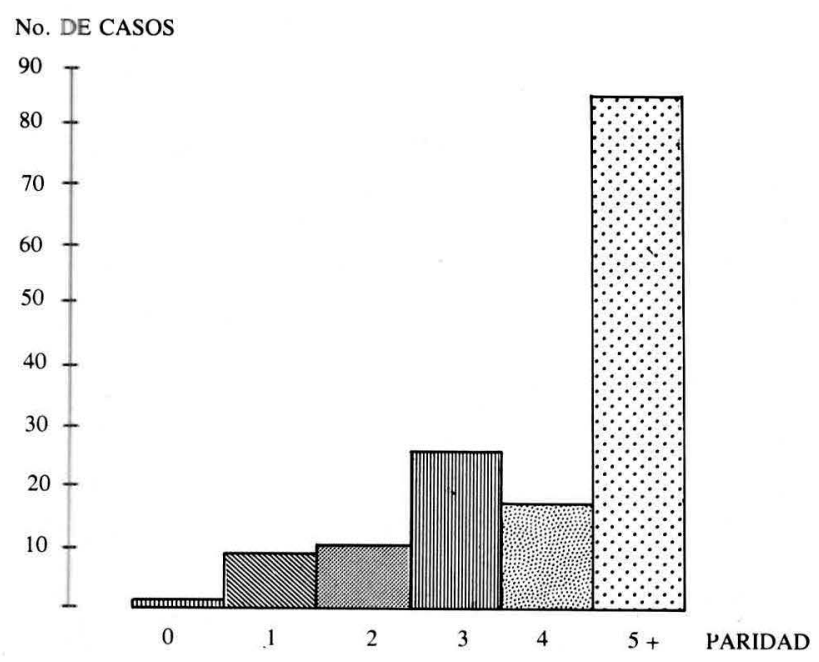

Cuadro 1

RESULTADO DEL CEC (SEGUN POSITIVIDAD)

\begin{tabular}{|ccc|}
\hline CEC & No. & $\%$ \\
\hline+ & 83 & 57.2 \\
- & 62 & 42.8 \\
\hline TOTAL & 145 & 100.0 \\
\hline
\end{tabular}

Cuadro 2

RESULTADO DEL CEC FRENTE A LA CITOLOGIA PARA CELULAS ESCAMOSAS

\begin{tabular}{|ccccccc|}
\hline & \multicolumn{5}{c|}{ Citología (Células Escamosas) } \\
CEC & No. & $\%$ & No. $^{(+)}$ & $\%$ & No. & Total \\
\hline$(-)$ & 6 & 4.2 & 56 & 38.9 & 62 & 43.1 \\
$(+)$ & 5 & 3.5 & 77 & 53.4 & 82 & 56.9 \\
\hline TOTAL & 11 & 7.7 & 133 & 92.3 & 144 & 100.0 \\
\hline
\end{tabular}

Cuadro 3

RESULTADO DEL CEC FRENTE A LA CITOLOGIA PARA CELULAS ENDOCERVICALES

\begin{tabular}{|cllllll|}
\hline CEC & \multicolumn{6}{c|}{ Citología (Células Endocervicales) } \\
& No. & $\%$ & No. & $\%$ & No. & $\%$ \\
\hline$(-)$ & 31 & 50 & 2 & 3.2 & 33 & 53.2 \\
$(+)$ & 27 & 43.5 & 2 & 3.2 & 29 & 46.7 \\
\hline TOTAL & 58 & 93.5 & 4 & 6.4 & 62 & 100.0 \\
\hline
\end{tabular}

Comparación del resultado del CEC frente a la biopsia exocervical: La distribución de los casos según la colposcopia fue:

Colposcopia insatisfactoria: 33 casos $(23 \%)$

Colposcopia satisfactoria: 109 casos $(77 \%)$

- Comparación del resultado de la biopsia exocervical y el $\mathrm{CEC}$ en casos con colposcopia insatisfactoria:

En colposcopia insatisfactoria el resultado del CEC frente a la biopsia exocervical, por grupos de enfermedad, se expone en el Cuadro 4.

Es de notar que en los casos con colposcopia insatisfactoria el $60 \%$ tenía un CEC positivo.

Comparando el resultado del CEC con la biopsia, en colposcopia insatisfactoria, según si la lesión fue mayor, igual o menor que en la biopsia, tenemos:

$\begin{array}{lrr} & \text { No. } & \% \\ \text { CEC }=\text { Biopsia } & 19 & 58 \\ \text { CEC }>\text { Biopsia } & 2 & 6 \\ \text { CEC }<\text { Biopsia } & 12 & 36\end{array}$

- Comparación del resultado de la biopsia exocervical y el CEC en casos con colposcopia satisfactoria:

En casos con colposcopia satisfactoria la comparación entre biopsia exocervical y resultado de CEC, por grupos de enfermedad, se expone en el Cuadro 5.

El 55\% de casos con colposcopia satisfactoria tiene CEC positivo.

Comparando el grado de lesión obtenido en el CEC y la biopsia, en casos con colposcopia satisfactoria, tenemos:

$\begin{array}{lrr} & \text { No. } & \% \\ \text { CEC }=\text { Biopsia } & 50 & 46 \\ \text { CEC }>\text { Biopsia } & 3 & 3 \\ \text { CEC }<\text { Biopsia } & 56 & 51\end{array}$

- Comparación del grado de lesión encontrada en el CEC frente a la biopsia exocervical:

La comparación del grado de lesión obtenido por los dos métodos en todos los casos es:

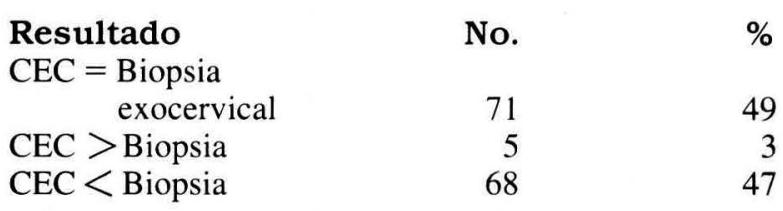

Comparación del resultado del CEC con el resultado final de anatomía patológica (AP): Los tratamientos definitivos realizados en este período fueron:

$\begin{array}{lrr}\text { Tratamiento } & \text { No. } & \% \\ \text { Conos } & 82 & 57 \\ \text { Histerectomía } & 63 & 43 \\ \text { TOTAL } & 145 & 100\end{array}$

- Comparación del resultado del CEC frente a AP final en previa colposcopia insatisfactoria:

En colposcopia insatisfactoria, comparando por grupos de lesión tenemos: Cuadro 6. 
Cuadro 4

COMPARACION DEL RESULTADO DE LA BIOPSIA EXOCERVICAL Y EL CEC EN LOS CASOS CON COLPOSCOPIA INSATISFACTORIA

\begin{tabular}{|c|c|c|c|c|c|c|c|}
\hline \multirow{3}{*}{ CEC } & \multirow{3}{*}{$(-)$} & \multicolumn{6}{|c|}{ Biopsia con Colposcopia Insatisfactoria } \\
\hline & & \multirow{2}{*}{ NICI } & \multirow{2}{*}{ I NICI } & \multirow{2}{*}{\multicolumn{2}{|c|}{ I NIC III Microinvasivo }} & \multicolumn{2}{|c|}{ Total } \\
\hline & & & & & & No. & $\%$ \\
\hline$(-)$ & 2 & 1 & 1 & 9 & - & 13 & 39.4 \\
\hline $\mathrm{NICI}$ & 1 & - & - & - & - & 1 & 3.0 \\
\hline NIC II & - & - & - & - & - & - & - \\
\hline NIC III & - & - & - & 17 & 1 & 18 & 54.5 \\
\hline Microinv. & - & - & - & 1 & - & 1 & 3.0 \\
\hline TOTAL $(\%)$ & $3(9.1)$ & $1(3.0)$ & $1(3.0)$ & $27(81.8)$ & $1(3.0)$ & 33 & 100.0 \\
\hline
\end{tabular}

Cuadro 5

COMPARACION DEL RESULTADO DE LA BIOPSIA EXOCERVICAL Y EL CEC EN LOS CASOS CON COLPOSCOPIA SATISFACTORIA

\begin{tabular}{|c|c|c|c|c|c|c|c|c|}
\hline \multirow{2}{*}{ CEC } & \multicolumn{8}{|c|}{ Biopsia con Colposcopia Satisfactoria } \\
\hline & $(-)$ & $\mathrm{NICI}$ & NICII & NIC III & Microinv. & Invas. & $\begin{array}{l}\text { Tota } \\
\text { No. }\end{array}$ & al \\
\hline$(-)$ & 2 & 2 & 1 & 42 & 2 & - & 49 & 44.9 \\
\hline $\mathrm{NICI}$ & - & 1 & 2 & 2 & - & - & 5 & 4.6 \\
\hline NIC II & 1 & - & - & 4 & - & - & 5 & 4.6 \\
\hline NIC III & 1 & - & 1 & 47 & - & 1 & 50 & 45.9 \\
\hline TOTAL $(\%)$ & $4(3.7)$ & $3(2.8)$ & $4(3.7)$ & $95(87.1)$ & $2(1.8)$ & $1(0.9)$ & 109 & 100.0 \\
\hline
\end{tabular}

Comparando la magnitud de la lesión encontrada en el CEC frente a la pieza final de patología, en pacientes con colposcopia insatisfactoria, tenemos:

$\begin{array}{lrr}\mathrm{CEC}=\mathrm{AP} & 16 & 49 \\ \mathrm{CEC}>\mathrm{AP} & 3 & 9 \\ \mathrm{CEC}<\mathrm{AP} & 14 & 42\end{array}$

- Comparación del resultado del CEC con AP de pieza quirúrgica en pacientes con previa colposcopia satisfactoria.

Esta comparación, de acuerdo al grado de lesión, la vemos en el Cuadro 7.

Comprando el grado de lesión del CEC con la pieza final, en pacientes con previa colposcopia satisfactoria, tenemos:

$\mathrm{CEC}=\mathrm{AP}$

$\mathrm{CEC}>\mathrm{AP}$

CEC $<$ AP
No.

54

8

63
$\%$

49

7

43
- Comparación del grado de lesión encontrado en el CEC frente a la pieza final en todos los casos:

\begin{tabular}{|c|c|}
\hline & No. \\
\hline $\begin{array}{l}\mathrm{CEC}=\mathrm{AP} \\
\mathrm{CEC}>\mathrm{AP}\end{array}$ & 71 \\
\hline $\mathrm{CEC}<\mathrm{AP}$ & $\begin{array}{l}11 \\
63\end{array}$ \\
\hline
\end{tabular}

Cuadro 6

COMPARACION DEL RESULTADO DEL CEC FRENTE AP FINAL EN PREVIA COLPOSCOPIA INSATISFACTORIA

\begin{tabular}{|cccccccc|}
\hline \multicolumn{6}{c}{ AP de Pieza Quirúrgica (Previa Colposcopia Insatisfactoria) } \\
& (-) & NICI & NICII & NICIII & Microinvas. & \multicolumn{2}{c|}{ Total } \\
& & & & & & No. & $\%$ \\
\hline NICI & 1 & 1 & & 10 & 1 & 13 & 39 \\
NIC III & 1 & & 1 & 15 & 1 & 1 & 3 \\
Microinv. & & & & 1 & & 1 & 35 \\
\hline TOTAL (\%) & $2(6)$ & $1(3)$ & $2(6)$ & $26(79)$ & $2(6)$ & 33 & 100 \\
\hline
\end{tabular}

Cuadro 7

COMPARACION DEL RESULTADO DEL CEC FRENTE AP FINAL EN PREVIA COLPOSCOPIA SATISFACTORIA

\begin{tabular}{|c|c|c|c|c|c|c|c|}
\hline \multirow{2}{*}{ CEC } & \multicolumn{7}{|c|}{ AP de Pieza Guirúrgica (Previa Colposcopia Satisfactoria) } \\
\hline & $(-)$ & $\mathrm{NICl}$ & NICII & NICIII & Invasivo & $\begin{array}{c}\text { Total } \\
\text { No. }\end{array}$ & $\%$ \\
\hline$(-)$ & 8 & 4 & 8 & 27 & 2 & 49 & 44.5 \\
\hline $\mathrm{NICl}$ & & 1 & & 3 & & 4 & 3.6 \\
\hline NICII & 2 & & 2 & 1 & 1 & 6 & 5.5 \\
\hline NIC III & 2 & & 4 & 43 & 2 & 51 & 46.4 \\
\hline TOTAL & $2(10.9)$ & $5(4.6)$ & $14(12.7$ & $74(67.3)$ & $5(4.5)$ & 100 & 100.0 \\
\hline
\end{tabular}

- Comparación del resultado del CEC en relación con la pieza final de patología (positiva o negativa):

La comparación de la positividad del CEC y la pieza final se describe en el Cuadro 8. Las pruebas de significancia del CEC, frente a la pieza final, nos demuestran:

No.

Sensibilidad

Especificidad

Valor predictivo +

Valor predictivo-

Falsos positivos

Falsos negativos

$\begin{array}{rr}\% & \\ 78 / 131 & 60 \\ 9 / 14 & 64 \\ 78 / 83 & 94 \\ 9 / 62 & 15 \\ 5 / 14 & 36 \\ 53 / 131 & 41\end{array}$

$\left(X^{2}=2.93\right.$ $\mathrm{p}<0.1)$

Estudio de la verdadera lesión en el canal endocervical y resultado del CEC: De los 145 casos, sólo en 60 la pieza de anatomía patológica reporta si existe o no lesión en el canal (41\%). 
Comparando el resultado de positividad del CEC frente a los casos con lesión positiva en el canal por anatomía patológica, encontramos lo expuesto en el Cuadro 9.

Se encontraron 11 casos de lesión en la unión escamocolumnar, que se toman como positivos de lesión en el canal de acuerdo con la descripción de patología.

En los casos que presentan CEC positivo para lesión, la pieza final no evidenció lesión en el canal en algunos, pero se reportó en la anatomía patológica lo siguiente:

1 caso: hiperplasia de células de reserva.

2 casos: metaplasia escamosa inmadura.

2 casos: hiperplasia de glándulas endocervicales.

Es de anotar que existen dos casos en que el CEC reportó adenocarcinoma de endocervix.

Las pruebas de significancia para el CEC, con relación a la lesión en el canal, muestran:

$\begin{array}{lrr} & \text { No. } & \% \\ \text { Sensibilidad } & 23 / 36 & 64 \\ \text { Especificidad } & 15 / 24 & 63 \\ \text { Valor predictivo + } & 23 / 32 & 72 \\ \text { Valor predictivo- } & 15 / 28 & 54 \\ \text { Falsos positivos } & 9 / 24 & 38 \\ \text { Falsos negativos } & 13 / 36 & 36\end{array}$

$$
\left(\mathrm{X}^{2}=4.02 \ldots \ldots \ldots \ldots . \mathrm{p}<0.05\right)
$$

Cuadro 8

COMPARACION DE POSITIVIDAD DEL CEC Y PIEZA FINAL DE PATOLOGIA'

\begin{tabular}{|c|c|c|c|c|c|c|}
\hline \multirow{2}{*}{ CEC } & \multicolumn{2}{|c|}{$(+)$} & \multicolumn{2}{|c|}{$\begin{array}{l}\text { AP } \\
(-)\end{array}$} & \multicolumn{2}{|c|}{ Total } \\
\hline & No. & $\%$ & No. & $\%$ & No. & $\%$ \\
\hline$(t)$ & 78 & 53.8 & 5 & 3.4 & 83 & 57.2 \\
\hline$(-)$ & 53 & 36.5 & 9 & 6.2 & 62 & 42.7 \\
\hline TOTAL & 131 & 90.3 & 14 & 9.6 & 145 & 100.0 \\
\hline
\end{tabular}

Comparando la magnitud de la lesión encontrada en el CEC, con la lesión encontrada en el canal en la pieza final, tenemos (Cuadro 12):

$\begin{array}{lrr} & \text { No. } & \% \\ \mathrm{CEC}=\mathrm{AP} & 22 & 37 \\ \mathrm{CEC}>\mathrm{AP} & 11 & 18 \\ \mathrm{CEC}<\mathrm{AP} & 27 & 45\end{array}$

Comparación de los diferentes medios diagnósticos y la patología final: Comparando el resultado de la biopsia exocervical y del CEC con la pieza final de patología, según los diferentes grupos de lesión, encontramos lo expuesto en el Cuadro 10.

Comparación de positividad: En el Cuadro 11 se compara la positividad para neoplasia encontrada por nuestro estudio en la biopsia exocervical y el CEC, frente a la pieza final de patología.
Cuadro 9

POSITIVIDAD DEL CEC FRENTE A POSITIVIDAD

DE LESION EN EL CANAL EN LA PIEZA FINAL DE ANATOMIA PATOLOGICA

\begin{tabular}{|c|c|c|c|c|c|c|}
\hline \multirow[t]{2}{*}{ CEC } & \multicolumn{6}{|c|}{$\begin{array}{l}\text { Lesión en Canal en AP Definitiva } \\
(+) \\
(-)\end{array}$} \\
\hline & No. & $\%$ & No. & $\%$ & No. & $\%$ \\
\hline$(+)$ & 23 & 38.3 & 9 & 15.0 & 32 & 53.3 \\
\hline$(-)$ & 13 & 21.7 & 15 & 25.0 & 28 & 46.7 \\
\hline TOTAL & 36 & 60.0 & 24 & 40.0 & 60 & 100.0 \\
\hline
\end{tabular}

Cuadro 10

COMPARACION DE LESIONES EN LA BIOPSIA

EXOCERVICAL, EL CEC Y LAS ENCONTRADAS EN LA PIEZA FINAL DE ANATOMIA PATOLOGICA

\begin{tabular}{|ccccccccccccccc|}
\hline & H & NICI & \multicolumn{1}{c|}{ NICI } & \multicolumn{1}{c|}{ NICIII } & \multicolumn{1}{c|}{ Microinvas. } & Invasivo & Total \\
\hline Biopsia & No. & $\%$ & No. & $\%$ & No. & $\%$ & No. & $\%$ & No. & $\%$ & No. & $\%$ & \\
Exocerv & 7 & 4.8 & 4 & 2.8 & 5 & 3.5 & 123 & 85.4 & 3 & 2.0 & 2 & 1.4 & 144 \\
AP & 14 & 9.7 & 6 & 4.1 & 16 & 11.0 & 100 & 69.0 & 2 & 1.4 & 7 & 4.8 & 145 \\
CEC & 62 & 42.8 & 5 & 3.4 & 6 & 4.1 & 70 & 48.3 & 1 & 0.7 & 1 & 0.7 & 145 \\
\hline
\end{tabular}

POSITIVIDAD PARA NEOPLASIA EN EL CEC, LA BIOPSIA Y LA PIEZA DE ANATOMLA PATOLOGICA

\begin{tabular}{|lrrrrr|}
\hline & No. $^{(+)}$ & No. $^{(-)}$ & $\%$ & $\begin{array}{c}\text { Total } \\
\text { No. }\end{array}$ \\
\hline Biopsia & 137 & 95 & 7 & 5 & 144 \\
AP & 131 & 90 & 14 & 10 & 145 \\
CEC & 83 & 57 & 62 & 43 & 145 \\
\hline
\end{tabular}

Cuadro 12

COMPARACION DEL GRADO DE LESION EN EL CANAL POR AP DEFINITIVA Y GRADO DE LESION ENCONTRADA EN EL CEC

\begin{tabular}{|c|c|c|c|c|c|c|c|}
\hline \multirow{2}{*}{ CEC } & \multirow{2}{*}{$(-)$} & \multicolumn{5}{|c|}{ Lesión en el Canal por AP Definitiva } & \\
\hline & & & & & & No. & $\%$ \\
\hline$(-)$ & 15 & 1 & 3 & 9 & & 28 & 46.7 \\
\hline $\mathrm{NICl}$ & & & 1 & & 1 & 17 & \\
\hline NICII & 2 & & & & & 2 & 3.3 \\
\hline NIC III & 7 & & 2 & 17 & 1 & 27 & 45.0 \\
\hline Invasivo & & & & & 2 & 2 & 3.3 \\
\hline TOTAL $(\%)$ & $24(40.0)$ & $1(1.7)$ & $6(10.0)$ & $26(43.3)$ & $3(5.0)$ & 60 & 100.0 \\
\hline
\end{tabular}

\section{Discusión}

La edad de las pacientes atendidas en la consulta de oncología oscila entre 20-75 años y encontramos que en el $82 \%$ de los casos corresponde a la edad entre 25-49 años, con una media de 39.7 años. Esto se correlaciona con otros estudios, donde la edad media encontrada fue de 32.9 años 
(16). La curva de la edad (Gráfica 1), corresponde a una distribución en campana, cuyo centro de mayor frecuencia está entre 35-39 años.

La gravidez, como parámetro epidemiológico indirecto, establece una distribución ascendente (Gráfica 2), encontrándose el mayor número de casos en las pacientes grávidas cuatro o más, con el $70 \%$.

Aunque la frecuencia de material insuficiente obtenida en el CEC es baja en los diferentes estudios, con un promedio del $17.1 \%$, nosotros encontramos un $28 \%$, debido posiblemente a que estas muestras se tomaron con curetaje de Novack o Radall, que no son las más apropiadas para el diagnóstico (7).

De los 62 casos en que se reporta el CEC como negativo, el $27 \%$ describía escaso material o fragmentos negativos. Suponemos que a mayor cantidad de material, aumentaría la positividad de la prueba (16).

En los casos con CEC positivo, las citologías positivas fueron el $92 \%$, encontrándose en mayor número de casos las citologías A3 con $60 \%$. de los casos.

De las pacientes con CEC positivo, y en las que la citología reportó células endocervicales $(43 \%)$, el $86 \%$ correspondió a citologías B2 y sólo un 7\% presentaba una patología mayor.

El porcentaje de colposcopia insatisfactoria fue de $23 \%$, mayor al que se describe en la literatura, que es hasta del $15-20 \%(3)$

Del total de la muestra (145 casos), el 57\% (83 casos) poseían un CEC positivo y de los casos con diagnóstico anatomopatológico definitivo adecuados (60 casos), se encontró 53\% (36 casos) con lesión en el canal.

De los 83 casos con CEC positivo, se realizó cono a 82 $(57 \%)$. Este mismo porcentaje se correlaciona muy bien con el número de lesiones halladas en el canal (53\%).

En pacientes con colposcopia satisfactoria, el CEC fue positivo en $55 \%$ de los casos, dato mucho mayor a lo reportado en la literatura, que es en promedio del $10 \%$. Esto se puede explicar en la mayoría de casos por contaminación de la muestra por una lesión exocervical, o también debido a que la lesión es multicéntrica, o que existen 2 lesiones diferentes.

En pacientes con esta misma colposcopia (satisfactoria), en CEC fue mayor que la lesión obtenida en la biopsia en sólo $3 \%$, que es bajo con relación a lo referido por otros estudios (promedio 6\%).

En pacientes con colposcopia insatisfactoria, el CEC fue positivo en $60 \%$ de los casos, mayor que el dado por otros estudios (promedio de 48\%).

En pacientes con colposcopia insatisfactoria, la lesión obtenida en el CEC fue mayor que la biopsia en $6 \%$, menor que lo encontrado por otros estudios (en promedio $17.5 \%$ ).

De todas maneras el porcentaje de lesión en el CEC igual a la biopsia exocervical es alto, tanto en pacientes con colposcopia insatisfactoria (58\%) como en pacientes con colposcopia satisfactoria ( $46 \%)$.

Frente al diagnóstico final por anatomía patológica (cono o histerectomía) el resultado obtenido en el CEC fue igual a la AP en $49 \%$ de casos con colposcopia insatisfactoria y en $49 \%$ de casos con colposcopia satisfactoria.

El resultado del CEC fue mayor que el obtenido por anatomía patológica en $9 \%$ de casos con colposcopia insa- tisfactoria y en $7 \%$ de casos con colposcopia satisfactoria. Esto probablemente se debe a la extracción de toda la lesión con el CEC.

Comparando todos los casos de CEC frente al resultado final de anatomía patológica, encontramos:

$\begin{array}{ll}\text { Sensibilidad } & 60 \% \\ \text { Especificidad } & 64 \% \\ \text { Valor predictivo + } & 94 \% \\ \text { Valor predictivo- } & 15 \%\end{array}$

Si comparamos el resultado del.CEC con la lesión en el canal, en comparación a otros estudios, tenemos:

$\begin{array}{lcc} & \text { Andersen(16) } & \text { Actual Estudio } \\ \text { Sensibilidad } & 55 \% & 64 \% \\ \text { Especificidad } & 75 & 63 \\ \text { Valor predictivo + } & 91 & 72 \\ \text { Valor predictivo- } & 27 & 54\end{array}$

En el presente estudio, comparando el resultado del CEC con la pieza final, los falsos (+) son de $36 \%$, y comparando el CEC con lesión endocervical, los falsos (+) son de $38 \%$, datos mucho mayores que los encontrados en la literatura (4 al 25\%). Creemos que estos falsos positivos se deben a contaminación, como lo demuestran los reportes de patología, donde es frecuente encontrar la descripción de "fragmentos con NIC...", que pueden corresponder a fragmentos de exocervix arrastrados en la muestra.

Comparando el CEC con el resultado de la pieza final de patología, encontramos un $41 \%$ de falsos negativos, y comparando el CEC con la lesión a nivel del canal, encontramos un porcentaje de falsos negativos de $36 \%$. Esto concuerda con la literatura, en donde se anotan falsos (-) entre 24 y $45 \%$ como lo refieren otros autores, esto se debe a la mala caliciad de la muestra, en la toma o en el proceso (16).

Con respecto al estudio de invasión, encontramos sólo 3 casos diagnosticados por el CEC, mientras el estudio de anatomía patológica nos revela 9 casos. Encontramos, pues, que el CEC no aporta al diagnóstico de invasión: dos pacientes con CEC negativo tenían invasión en la anatomía patológica definitiva, mientras las biopsias exocervicales mostraban micro-invasión en los mismos casos. En los dos casos la colposcopia fue positiva-satisfactoria.

Observando los 58 casos totales en los que la pieza final reveló lesión en el canal, anotamos:

- Biopsia de exocervix fue positiva en $95 \%$ de los casos.

- La colposcopia satisfactoria en $70 \%$ de los casos, insatisfactoria en $30 \%$ de los casos.

- El CEC fue positivo en $61 \%$ de los casos en que este fue apto para diagnóstico.

\section{Conclusiones}

1. El porcentaje de pacientes con CEC positivo fue mayor $(60 \%)$ en las pacientes que tenían colposcopia insatisfactoria, con relación a las que tenían colposcopia satisfactoria $(55 \%)$.

2. El CEC aumentó el diagnóstico dado sólo por la biopsia en $3.5 \%$ de los casos (CEC > Biopsia $=3.5$ ).

3. El CEC aumentó el diagnóstico dado sólo por la biopsia 
en $6 \%$ de los casos con colposcopia insatisfactoria (CEC

$>$ Biopsia $=6 \%$ ).

4. De acuerdo al siguiente esquema:

CEC vs. Diagnóstico Hịstológico de Lesión en el Canal

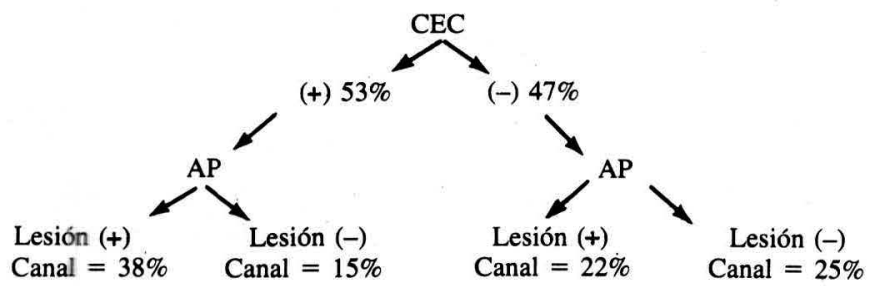

El porcentaje de resultados de lesión positiva en el canal, cuando el CEC es negativo (25\%), es menor que el porcentaje de resultados de lesión positiva en el canal cuando el CEC es positivo (38\%).

5. De acuerdo at siguiente esquema:

\section{CEC vs. Diagnóstico Anatomopatológico Definitivo}

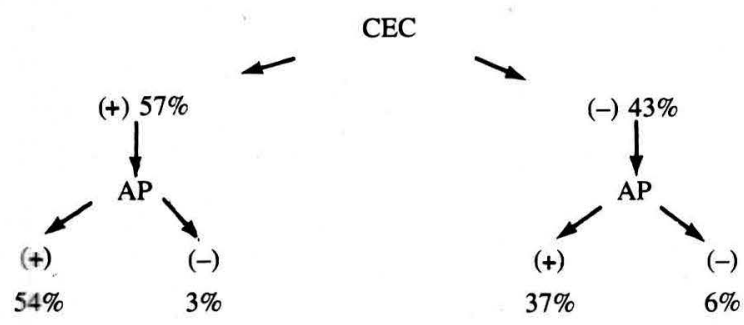

El porcentaje de resultados positivos en la pieza final es menor cuando el CEC es negativo (37\%), que cuando el CEC es positivo (54\%).

6. El porcentaje de positividad es mayor en cuanto mayor sea la muestra en cantidad (esto lo basamos en el $27 \%$ de casos en que se reporta CEC (-) con fragmentos o escaso material).

7. Con base en los datos obtenidos, concluimos que en nuestra institución se está realizando un número racional de conos, como lo demuestra en la correlación entre el CEC (+) (57\%), lesión positiva en el canal en la pieza final $(53 \%)$ y número de conos $(57 \%)$.

8. El CEC tiene gran importancia para localizar lesiones en el canal, como lo demuestra su alto valor predictivo positivo (94\%).

9. Con base en lo observado, constatamos que los falsos positivos se deben a contaminación de la muestra.

10. Los falsos negativos se reducirán en la medida que mejore la calidad de la muestra (tanto en la toma como en el proceso).

11. El CEC no ayuda al diagnóstico de invasión.

En resumen estamos de acuerdo con los criterios de Andersen (16), en cuanto a los beneficios del CEC, como son:

1. El CEC puede revelar una lesión más severa que en el exocervix.

2. El CEC ocasionalmente es el único test que aporta resultados anormales.

3. El CEC es necesario para la evaluación de posibles adenocarcinomas (dos casos en nuestro estudio).

- El CEC es, junto con la citología, la colposcopia y la biopsia, un método diagnóstico que ayuda a localizar la

Gráfica 3

FLUJOGRAMA DIAGNOSTICO PARA NEOPLASIA CERVICAL

(Shier R.M., De Petrillo D.U. Texas) (3)

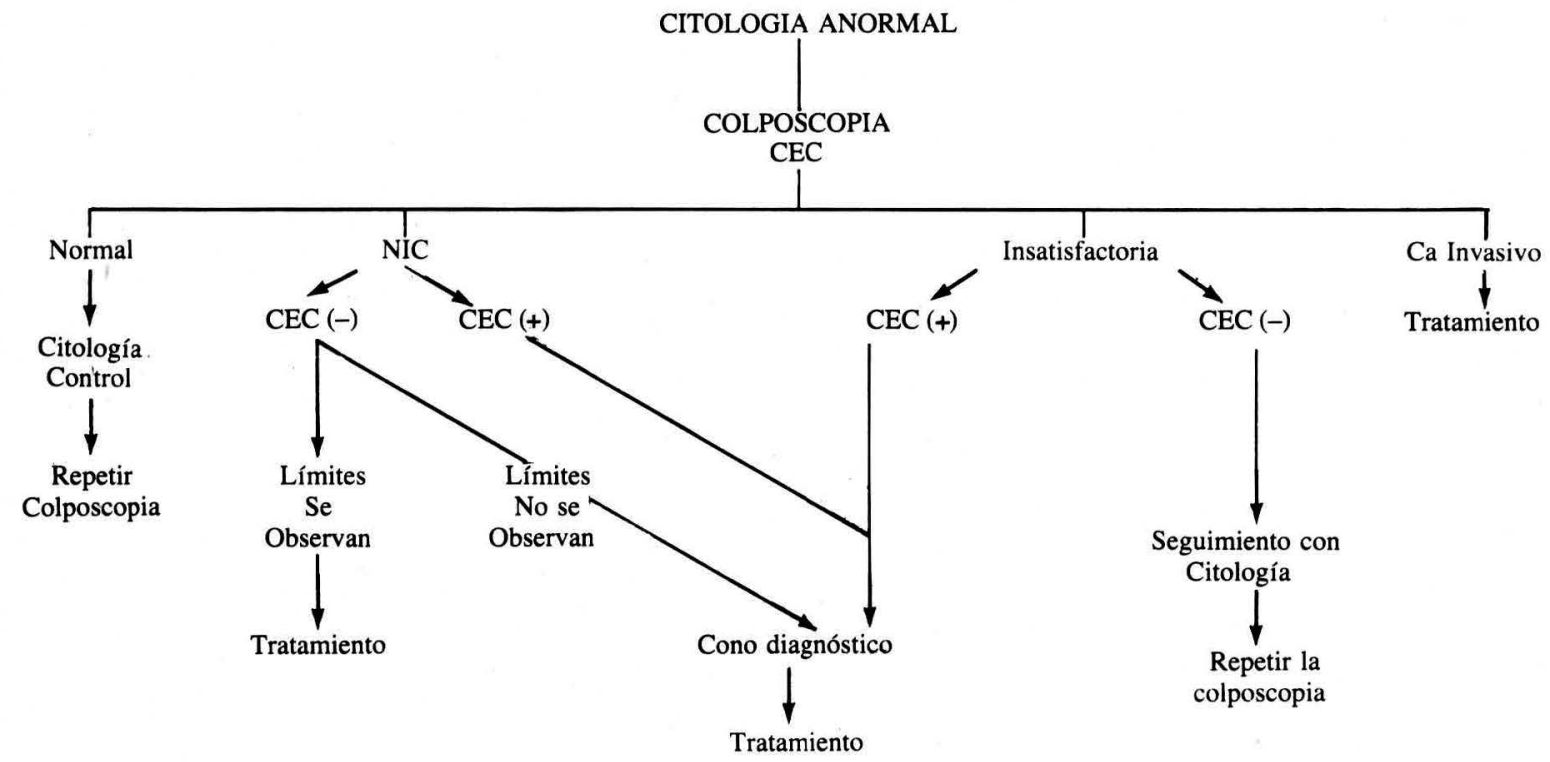

CEC: Curetaje endocervical. 
lesión endocervical y que correlacionado con los otros medios diagnósticos, obtendremos una apreciación más acertada acerca de la patología cervical.

- Dentro de este margen de ideas creemos que el CEC es un arma valiosa en pacientes con patología de cuello en las que se pretende realizar tratamiento conservador (ejemplo: crioterapia), como lo refiere Wetrich (13).

- Los resultados obtenidos dan base para modificar los esquemas de diagnóstico en el NIC, introduciendo más de lleno el CEC, comparable al flujograma diagnóstico de Shier y De Petrillo, de la Universidad de Texas (3), (Gráfica 3), con algunas modificaciones.

- Por último debemos tener en cuenta las palabras sabias de Hath (11), que dicen: "El CEC es considerado una más confiable evaluación del canal endocervical, que la simple observación por el ojo".

\section{BIBLIOGRAFIA}

1. Mattingly RF, Thompson JD. Ginecología Operatoria del Richard W. Telinde. Editorial El Ateneo S.A. (5a. ed.). Barcelona, 1977. p. 591.

2. Stafl A, Mattingly RF. Colposcopic Diagnosis of cervical Neoplasia. Obstet Gynecol 1973; 41: 168

3. Rutledge MD, Freedman RS, Gershenson DM. Gynecologic cancer. Diagnosis and treatment strategies. University of Texas press. Austin, 1985. p. 211.

4. Seiguer BK. Ginecología infanto-juvenil. Editorial Médica Panamericana (2a. ed.). Buenos Aires, 1987. p. 103.

5. Calatronic CJ, Ruiz V. Terapéutica Ginecológica. Editorial Médica Panamericana (9a. ed.). Buenos Aires, 1981. p. 639.

6. Benson RC. Diagnóstico y Tratamiento Ginecobstétricos. Editorial El Manuel Moderno (3a. ed.). México D.F., 1983. p. 229.

7. Manual de normas y procedimientos para el control del cáncer del cuello uterino. Serie Paltex. No. 6 OPS/OMS. 1985. p. 17.

8. Disaia PJ, Creasman WT. Clinical gynecologic oncology. The C. Mosby Company. St. Louis, Toronto, Princeton 1984 (2a. ed.). p. 13.

9. Jordam AJ. Colposcopia en el diagnóstico de carcinoma cervical y fase precancerosa. Ginecología y Obstetricia: Temas actuales 1985; 1: 75 .

10. Noumoff JS. Atipya in cervical cytology as a rish factor dor intraepithelial Neoplasia. Am J Obstet Gynecol 1987; 156: 628.

11. Hatch KD, Shingleton HM, Orr JW, Gore H, Soog S-J. Role of endocervical curettage in colposcopy. Obstet Gynecol 1985; 65: 403.

12. Moseley KR, Dihn TV, Hannigan EV, Dillar EA, Yandell RB. Ne- cessity of endocervical curettage in colposcopy. Am J Obstet Gynecol 1986; 154: 992

13. Wetrich DW. An analysis of the factors involved in the colposcopic evaluation of 2.194 patients with abnormal papanicolaou smears. Am J Obstet Gynecol 1986; 154: 1339.

14. Spirtos NM, Schlaerth JB, d'Ablaing III G, Morrow CP. A critical evaluation of the endocervical curettage. Obstet Gynecol 1987; 70: 729.

15. Donohue LR, Merewether W. Colposcopy in diagnosis of cervical Neoplasias. Am J Obstet Gynecol 1972; 113: 107.

16. Andersen W, Frierson H, Barber S, Tabbarah S, Taylor P, Underwood P. Sensitivity and specificit of endocervical curettage and the endocervical brush for the evaluation of the endocervical canal. Am J Obstet Gynecol 1988; 159: 702.

17. Dreschet CW, Peters WD, Roberts JA. Contribution of endocervical curettage in evaluating abnormal cervical cytology. Obstet Gynecol 1983; 62: 343 .

18. Swan RW. Evaluation of colposcopic accuracy without endocervical curettage. Obstet Gynecol 1979; 53: 680 .

19. Urcuyo R, Rome RM, Nelson JH. Some observations on the value of endocervical curettage performed ad an integral part of colposcopic examination of patients with abnormal cervical cytology. Am J Obstet Gynecol 1977; 128: 787 .

20. Rodrigo FM. La conización en el Instituto Materno Infantil, años 1985-1988. En: "Complicaciones médico-quirúrgicas en Ginecología: Experiencia institucional. 1989. p. 75.

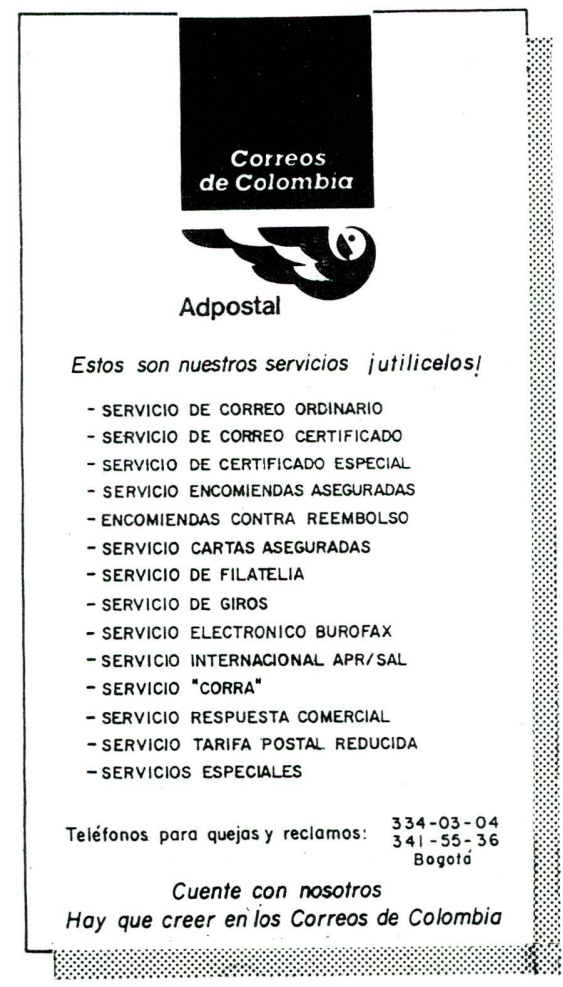

